\title{
Naringin attenuates rat myocardial ischemia/reperfusion injury via PI3K/Akt pathway-mediated inhibition of apoptosis, oxidative stress and autophagy
}

\author{
FENGWEI LI, ZHENJIAN ZHAN, JIN QIAN, CHUANBIN CAO, WEI YAO and NENG WANG \\ Department of Cardiology, Suizhou Hospital, Hubei University of Medicine, Suizhou, Hubei 441300, P.R. China
}

Received November 6, 2019; Accepted March 17, 2021

DOI: $10.3892 /$ etm.2021.10243

\begin{abstract}
Naringin (NRG) has been reported to exert cardioprotective effects against multiple cardiovascular diseases, including lipopolysaccharide-induced and hyperglycemia-induced myocardial injury. However, the role of NRG in myocardial ischemia/reperfusion (I/R) injury remains unclear. In the present study, the PI3K/Akt pathway was investigated to evaluate the possible mechanisms underlying the roles of NRG in myocardial ischemia/reperfusion (I/R) injury. The levels of cardiac enzymes were measured by ELISA to evaluate the optimal dosage of NRG that could protect against myocardial I/R injury. Rats were administered $100 \mathrm{mg} / \mathrm{kg}$ of NRG and activities of myocardial enzymes, the level of cardiac apoptosis and inflammation, oxidant response, autophagy indicators and echocardiography were evaluated. The level of corresponding proteins was measured using western blotting. The results indicated that NRG elicited the best cardioprotective effects at a dose of $100 \mathrm{mg} / \mathrm{kg}$ by significantly reducing the levels of myocardial enzymes, apoptosis, inflammation, oxidative response and infarct size. Furthermore, NRG alleviated contractile dysfunction by increasing the left ventricular ejection fraction and fractional shortening. In addition, NRG markedly promoted the phosphorylation of Akt, while decreasing the level of autophagy indicator beclin-1 and the microtubule-associated protein 1B-light chain 3 (LC3B) II/ LC3BI ratio. However, PI3K/Akt inhibitor (LY294002) partially reduced the NRG induced phosphorylation of $\mathrm{Akt}$ and the reduction in beclin-1, along with the LC3BII/LC3BI ratio. The results of the present study demonstrated that NRG could attenuate myocardial I/R injury.
\end{abstract}

Correspondence to: Dr Neng Wang, Department of Cardiology, Suizhou Hospital, Hubei University of Medicine, 60 Longmen Road, Suizhou, Hubei 441300, P.R. China

E-mail: szhxnknwang@163.com

Key words: naringin, myocardial ischemia/reperfusion injury, PI3K/Akt, apoptosis, oxidative stress, autophagy

\section{Introduction}

Acute myocardial infarction (AMI) is a life-threatening disease characterized by high morbidity and mortality (1). Ischemia/reperfusion ( $\mathrm{I} / \mathrm{R})$ injury is often accompanied by the restoration of occluded arteries, leading to excessive inflammation, oxidative stress and cardiomyocyte apoptosis $(2,3)$. Furthermore, I/R-mediated myocardial damage increases the mortality of patients with AMI (4). Therefore, it is urgent to develop effective therapeutic strategies for the prevention of I/R injury.

Naringin (NRG), a citrus flavonoid, was initially extracted from pomelo peel (5). In traditional Chinese medicine, NRG is reported to exert protective effects against multiple cardiovascular diseases. Chen et al (6) demonstrated that NRG could alleviate anoxia/reoxygenation induced cardiomyocyte apoptosis by promoting nuclear factor erythroid 2-related factor 2 (Nrf2) nuclear translocation in vitro. Jian et al (5) indicated that NRG was able to relieve doxorubicin-induced cardiotoxicity by suppressing myocardial apoptosis via the p38MAPK pathway. Additionally, NRG was observed to attenuate sepsis-associated cardiac dysfunction by inhibiting the inflammatory response and oxidative stress (7). Though these studies indicate that NRG may be useful as a therapeutic agent in the treatment of cardiovascular diseases, its effects on myocardial I/R injury require further investigation.

The PI3K/Akt signaling pathway is a conserved family of signal transduction pathway that participates in the modulation of multiple biological processes, including cell proliferation, oxidative response and cardiac apoptosis (8). Numerous studies have demonstrated that pharmacological activation of the PI3K/Akt pathway could effectively enhance the defensive ability of cardiomyocytes against I/R injury, as manifested by an increase in anti-inflammatory, anti-apoptotic and anti-oxidative capacity $(9,10)$. In addition, the PI3K/Akt signaling pathway was reported to depress I/R-induced excessive autophagy thus attenuating I/R injury $(11,12)$. A study by Rani et al (13) also suggested that the cardioprotective effects of NRG were closely related to the PI3K/Akt pathway.

In the present study whether NRG could alleviate myocardial I/R injury in vivo was investigated. In addition, the in-depth modulatory effect of NRG on the PI3K/Akt signaling cascade was examined. 


\section{Materials and methods}

Animals. All procedures involving animals were conducted according to the Guide for the Care and Use of Laboratory Animals (NIH Publication no. 85-23, revised 1996) (14) and were approved by the Institutional Animal Care and Use Committee of Hubei University of Medicine. A total of 80 male Sprague-Dawley rats (weight 210-250 g; age, 7-8 weeks) were purchased from Hubei University of Medicine. The rats were acclimatized to the specific pathogen free conditions at a room temperature $24 \pm 2^{\circ} \mathrm{C}$, humidity of $55 \pm 5 \%$ on a 12 -h light/dark cycle and were allowed free accesses to food and water.

$I / R$ model and protocol for in vivo experiments. An I/R model was established as previously described (12). Briefly, rats were anesthetized by intraperitoneal injection of sodium thiopentone $(70 \mathrm{mg} / \mathrm{kg})$ and then mechanically ventilated with tracheal intubation (70 strokes/min). Subsequently, thoracotomy and pericardiotomy were performed at the left side in the three to four intercostal space to expose the heart. The left anterior descending (LAD) coronary artery was occluded using a 6-0 silk suture 2-3 mm below the left auricle. An obvious elevation in the ST segment was considered as successful establishment of ischemia. After $30 \mathrm{~min}$ of LAD occlusion, the artery was reperfused for $180 \mathrm{~min}$. NRG (HPLC $\geq 99 \%$; Shanghai Winherb Medical S\&T Development Co., Ltd.) was dissolved in normal saline (15) and continuously administrated by intraperitoneal injection for 1 week before I/R induction. LY294002 (PI3K/Akt inhibitor, $0.3 \mathrm{mg} / \mathrm{kg}$; MedChemExpress) was given $30 \mathrm{~min}$ before I/R induction via caudal vein injection.

Rats were first randomly assigned to the following groups to determine the optimal dose of NRG administration: i) Sham group; ii) I/R group; iii) NRG $(25 \mathrm{mg} / \mathrm{kg} / \mathrm{day})+\mathrm{I} / \mathrm{R}$ group (NRG25 + I/R); iv) NRG $(50 \mathrm{mg} / \mathrm{kg} /$ day $)+\mathrm{I} / \mathrm{R}$ group (NRG50 + I/R); and v) NRG (100 mg/kg/day) + I/R group $(\mathrm{NRG100}+\mathrm{I} / \mathrm{R})$. The $100 \mathrm{mg} / \mathrm{kg}$ dose of NRG was proven to exert the best cardioprotective effects and subsequently the rats were randomly assigned into the following groups to evaluate the functions of NRG in myocardial I/R injury: i) Sham group; ii) I/R group; iii) NRG100 + I/R group; and iv) NRG100 + I/R + LY294002 group (NRG100 + I/R + LY).

Evaluation of myocardial injury. After 180 min of reperfusion, blood specimens were obtained and centrifuged $1,000 \mathrm{x} \mathrm{g}$ for $15 \mathrm{~min}$ at a room temperature in preparation for the measurement of creatine kinase (CK), lactate dehydrogenase $(\mathrm{LDH})$ and cardiac troponin I (cTnI). Creatine kinase isoenzyme Assay kit (cat. no. E006; Nanjing Jiancheng Bioengineering Institute), lactate dehydrogenase assay kit (cat. no. A020-1; Nanjing Jiancheng Bioengineering Institute) and Troponin Assay kit (no. E019-1; Nanjing Jiancheng Bioengineering Institute) were used for the analyses according to the manufacturer's instructions.

Assessment of infarct size. 2,3,5-triphenyltetrazolium chloride (TTC; Sigma-Aldrich; Merck KGaA) staining was applied to evaluate the infarcted size as previously described (8). Hearts were harvested and frozen at $-80^{\circ} \mathrm{C}$ after 180 min of reperfusion. Subsequently, hearts were cut from the apex to the base, these slices were then incubated with $1 \%$ TTC for $20 \mathrm{~min}$ at $37^{\circ} \mathrm{C}$. The infarct area (white) were evaluated using Image Pro Plus 6.0 (Media Cybernetics, Inc.).

Assessment of oxidative stress. Blood specimens were obtained for the measurement of malondialdehyde (MDA) and superoxide dismutase (SOD) using Malondialdehyde assay kit (cat. no. A003-1; Nanjing Jiancheng Bioengineering Institute) and Superoxide Dismutase assay kit (cat. no. A001-3; Nanjing Jiancheng Bioengineering Institute) according to the manufacturer's instructions.

Detection of myocardial apoptosis. The degree of myocardial apoptosis was assessed by terminal deoxynucleotidyl transferase dUTP nick end labeling (TUNEL) staining using the In Situ Cell Death Detection kit (cat. no. 11684817910; Roche Diagnostics $\mathrm{GmBH}$ ) in accordance with the manufacturer's instructions. Cardiomyocytes with marked nuclear labeling were considered TUNEL-positive using a fluorescence microscope. A total of 3 fields (magnification, x200) were randomly selected per heart to determine apoptosis index (AI) using image-pro 5.0 (Media Cybernetics, Inc.). The formula for calculating $\mathrm{AI}$ was as follows, $\mathrm{AI}=$ number of apoptotic cells/total number of cells counted.

Analysis of the inflammatory response. The levels of interleukin-1 $\beta$ (IL-1 $\beta$ ), interleukin-6 (IL-6) and tumor necrosis factor- $\alpha($ TNF- $\alpha$ ) released from myocardium were evaluated using Interleukin-1 $\beta$ Assay kit (cat. no. H002; Nanjing Jiancheng Bioengineering Institute), Interleukin-6 Assay kit (cat. no. H007; Nanjing Jiancheng Bioengineering Institute) and Tumor Necrosis Factor- $\alpha$ Assay kit (cat. no. H052; Nanjing Jiancheng Bioengineering Institute) in accordance with the manufacturer's instructions.

Histopathological examination. Heart samples were fixed in paraffin and cut into transverse sections $(5-\mu \mathrm{m}$ thick) before staining with hematoxylin and eosin (H\&E) (16). The stained slices were then analyzed under a light microscope (model no. BX51; Olympus Corporation). A total of 5 fields (magnification, x200) were randomly chosen from each group and scored in a blinded manner by 2 individuals (Department of Cardiology, Suizhou Hospital) according to the following criteria as previously described (17): 0 , no abnormality was observed; 0.5, with minor lesions; 1.0 , with sparse lesions; 1.5, with intermediate lesions; and 2.0, extensive hemorrhage and leukocytic infiltration.

Echocardiography. Cardiac performance was analyzed by non-invasive echocardiography after a $180 \mathrm{~min}$ period of reperfusion using an ultrasound Doppler imaging system [Vinno Technology (Suzhou) Co., Ltd.]. The heart rate (HR), left ventricular internal dimension systole (LVIDs), left ventricular internal dimension diastole (LVIDd), ejection fraction (EF) and fractional shortening (FS) were obtained as previously described (18).

Western blotting. Myocardial tissue was immediately harvested and stored in liquid nitrogen $180 \mathrm{~min}$ after reperfusion. Western blotting was carried out using a previously described 

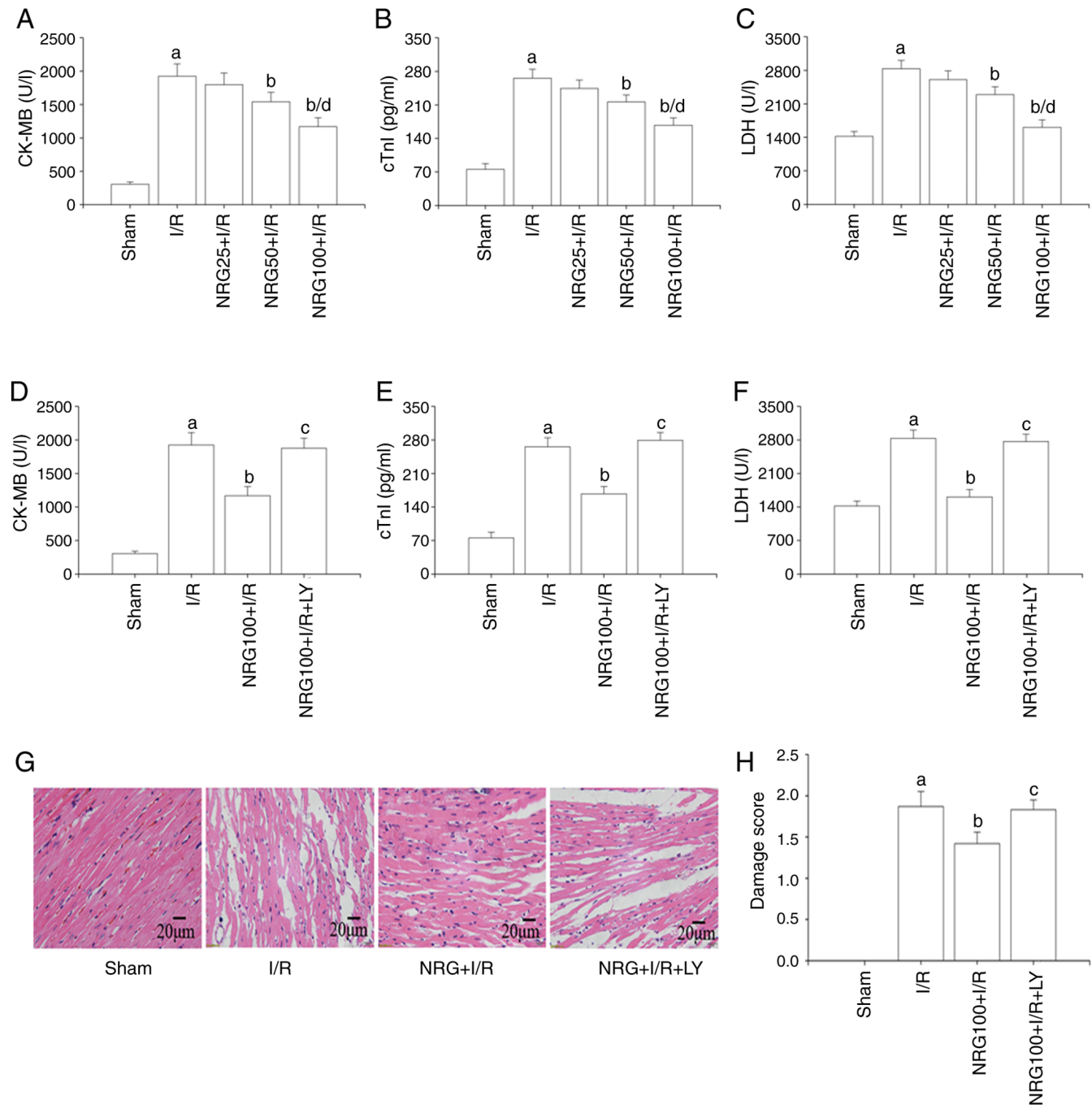

Figure 1. NRG pretreatment attenuates cardiac I/R injury. Serum levels of (A) CK-MB, (B) cTnI and (C) LDH (n=5) after I/R and following pretreatment with different NRG doses. The expression levels of (D) CK-MB, (E) cTnI and (F) LDH (n=5) after I/R with use of a PI3K/Akt inhibitor. (G) Representative images of heart tissues following hematoxylin and eosin staining. (H) Damage score $(n=5)$. NRG, naringin; I/R, ischemia reperfusion; CK-MB, creatine kinase myocardial band; cTn1, cardiac troponin 1; LDH, lactate dehydrogenase; LY, PI3K/Akt inhibitor LY294002; NRG25, 25 mg/kg naringin; NRG50, 50 mg/kg naringin; NRG100, $100 \mathrm{mg} / \mathrm{kg}$ naringin. ${ }^{\mathrm{a}} \mathrm{P}<0.05$ vs. sham group; ${ }^{\mathrm{b}} \mathrm{P}<0.05$ vs. $\mathrm{I} / \mathrm{R}$ group; ${ }^{\mathrm{C}} \mathrm{P}<0.05$ vs. I/R group; ${ }^{\mathrm{d}} \mathrm{P}<0.05$ vs. NRG50 + I/R group.

method (19). Primary antibodies applied for western blotting were as follows: Bcl-2 (1:2,000; cat. no. ab59348; Abcam); p-Akt (1:1,000, cat. no. 4060, Cell Signaling Technology, USA); Akt (1:3000, cat. no. 4691, Cell Signaling Technology, Inc.); GADH (1:10,000; cat. no. ab37168; Abcam); cleaved caspase 3 (1: 1,000; cat. no. AF7022, Affinity Biosciences Ltd.); microtubule-associated protein 1B-light chain 3 (LC3BI; 1:1,000, ; cat. no. 4599; Cell Signaling Technology, Inc.); LC3BII (1:1,000; cat. no. 3868; Cell Signaling Technology, Inc.); beclin-1 (1:2,000; cat. no. 3495; Cell Signaling Technology, Inc.) and caspase 3 (1:1,000; cat. no. ab44976, Abcam).

Secondary antibody was horseradish peroxidase (HRP) goat anti-Rabbit (1:10,000; cat. no. AS1107; Wuhan Aspen Biotechnology Co. Ltd.)

Statistical analysis. Continuous variables are presented as the mean \pm standard deviation (SD) and were analyzed using SPSS 18.0 (SPSS, Inc.). One-way analysis of variance (ANOVA) followed by Tukey's post hoc test was used for multiple comparisons among groups. $\mathrm{P}<0.05$ was defined as statistically significant.

\section{Results}

$N R G$ administration alleviates myocardial $I / R$ injury. The level of myocardial enzymes can reflect the degree of I/R-induced myocardial damage (20). As demonstrated in Fig. 1A-C, I/R significantly promoted the release of the cardiac enzymes CK-MB, cTnI and LDH in comparison with sham surgery, while pretreatment with NRG decreased the levels of these myocardial enzymes released following I/R. NRG pretreatment at a dose of $100 \mathrm{mg} / \mathrm{kg}$ displayed the highest cardioprotective effect against I/R injury, as indicated by the significant reduction in the levels of creatine kinase myocardial band (CK-MB), cTnI and LDH as compared to I/R. Therefore, the optimal dose of NRG was considered to be $100 \mathrm{mg} / \mathrm{kg}$. The results also indicated that pretreatment with LY294002, a PI3K/Akt inhibitor, reversed the cardioprotective effects of NRG (Fig. 1D-F). The myocardial structure in the I/R group 
A

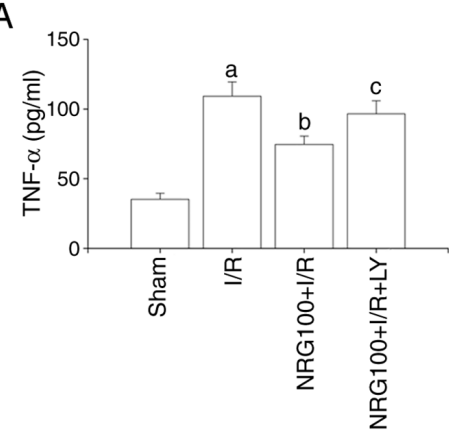

D

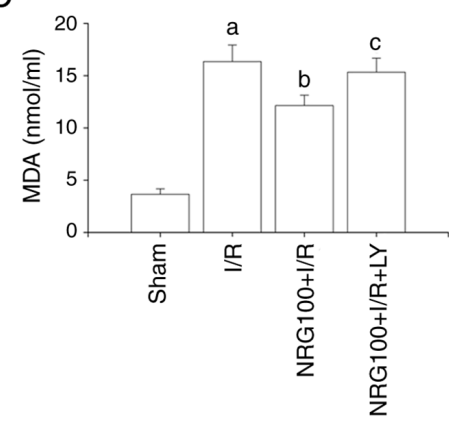

B

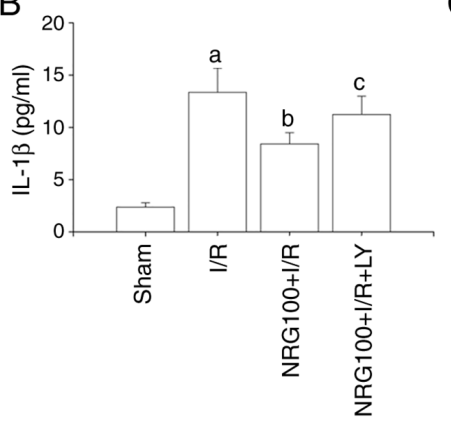

E

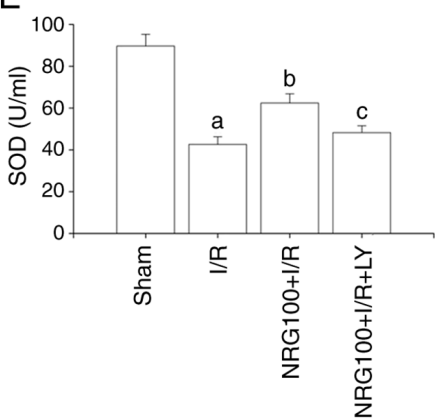

G

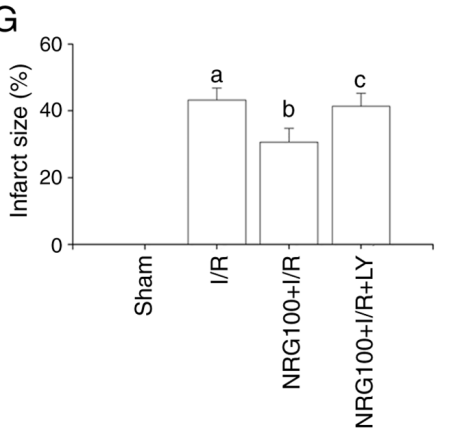

C

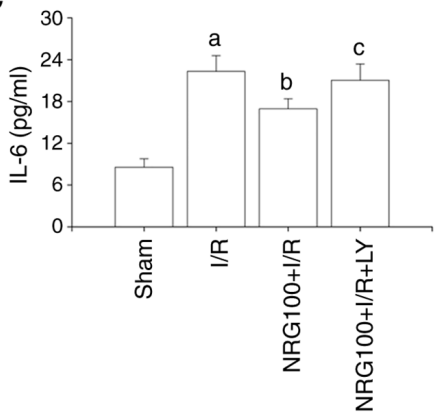

$\mathrm{F}$

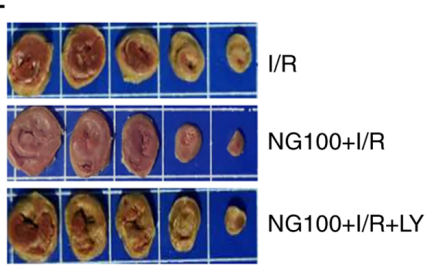

Figure 2. NRG pretreatment alleviates I/R-accelerated inflammatory reactions, oxidative stress and infarct size. The expression levels of (A) TNF- $\alpha$, (B) IL-1 $\beta$ and (C) IL-6 (n=5); (D) The level of MDA ( $n=5)$. (E) The level of SOD ( $=5)$. (F) Representative images of 2,3,5-triphenyltetrazolium chloride stain. (G) Quantitative analysis of infarct size $(n=3)$. NRG, naringin; I/R, ischemia reperfusion; TNF- $\alpha$, tumor necrosis factor- $\alpha$; IL, interleukin; MDA, malondialdehyde; SOD, superoxide dismutase; NRG100, $100 \mathrm{mg} / \mathrm{kg}$ naringin; LY, PI3K/Akt inhibitor LY294002. ${ }^{\text {P }}<0.05 \mathrm{vs}$. sham group; ${ }^{\text {P }}<0.05 \mathrm{vs}$. I/R group; ${ }^{\mathrm{C}} \mathrm{P}<0.05$ vs. NRG100 + I/R group.

was altered in comparison with that in the sham animals with a disorderly arrangement of cardiomyocytes, necrosis and hemorrhage and relatively high damage scores (Fig. 1G-H). However, I/R-induced myocardial structural changes were significantly reduced by NRG treatment with a lower damage score, while LY294002 abolished the effects of NRG.

Anti-inflammatory and anti-oxidative effects of NRG. The results of the present study suggested that I/R triggered an inflammatory response by increasing the release of inflammatory factors TNF- $\alpha$, IL-1 $\beta$ and IL-6 (Fig. 2A-C). $\mathrm{NRG}$ pretreatment significantly inhibited the release of inflammatory factors accelerated by I/R. However, LY294002 partially abrogated the effects of NRG.

A significant reduction in SOD activity and MDA concentration was observed in the $\mathrm{NRG}+\mathrm{I} / \mathrm{R}$ group in comparison with I/R (Fig. 2D-E). However, the effects of NRG were abolished by LY294002. These finding demonstrated that NRG could mitigate I/R-induced oxidative stress and inflammatory signaling.
Assessment of infarct size. As demonstrated in Fig. 2F and G, the infarct size in the I/R group was significantly increased in comparison with the sham group. However, NRG pretreatment notably reduced the infarct size and these effects were abrogated by LY294002.

Effects of NRG on heart function. As displayed in Table I, I/R significantly aggravated cardiac dysfunction by enlarging LVIDs and impairing EF and FS. However, NRG pretreatment markedly improved cardiac performance by narrowing LVIDs while promoting FS. The administration of the PI3K/Akt inhibitor LY294002, abrogated the effects of NRG.

$N R G$ pretreatment reduces myocardial apoptosis. The present results showed that the AI (Fig. 3A-B) and the expression of cleaved caspase 3 were dramatically increased in the I/R group, while the level of Bcl-2 was notably decreased in comparison with the sham group (Fig. 4A-D). NRG pretreatment markedly reduced $\mathrm{AI}$ and cleaved caspase 3 expression and significantly increased the level of $\mathrm{Bcl}-2$ in comparison with the I/R group. 
Table I. Effects of NRG on cardiac performance.

\begin{tabular}{|c|c|c|c|c|c|}
\hline Group & $\begin{array}{c}\text { HR } \\
\text { (beats/min) }\end{array}$ & $\begin{array}{l}\text { LVIDs } \\
(\mathrm{mm})\end{array}$ & $\begin{array}{l}\text { LVIDd } \\
(\mathrm{mm})\end{array}$ & $\begin{array}{l}\mathrm{EF} \\
(\%)\end{array}$ & $\begin{array}{l}\text { FS } \\
(\%)\end{array}$ \\
\hline Sham & $442.32 \pm 16.32$ & $2.41 \pm 0.31$ & $4.98 \pm 0.18$ & $84.62 \pm 3.21$ & $59.48 \pm 2.69$ \\
\hline $\mathrm{I} / \mathrm{R}$ & $429.85 \pm 15.75$ & $3.24 \pm 0.36^{\mathrm{a}}$ & $5.02 \pm 0.21$ & $55.36 \pm 4.23^{\mathrm{a}}$ & $32.25 \pm 1.38^{\mathrm{a}}$ \\
\hline $\mathrm{NRG} 100+\mathrm{I} / \mathrm{R}$ & $432.48 \pm 17.63$ & $2.95 \pm 0.25^{b}$ & $5.07 \pm 0.23$ & $66.31 \pm 2.41^{\mathrm{a}}$ & $37.62 \pm 1.57^{\mathrm{a}}$ \\
\hline $\mathrm{NRG} 100+\mathrm{I} / \mathrm{R}+\mathrm{LY}$ & $425.17 \pm 19.32$ & $3.19 \pm 0.32^{c}$ & $5.04 \pm 0.20$ & $53.36 \pm 4.06^{\mathrm{a}}$ & $31.24 \pm 1.45^{\mathrm{a}}$ \\
\hline
\end{tabular}

HR, heart rate; LVIDs, left ventricular internal dimension systole; LVIDd, left ventricular internal dimension diastole; EF, ejection fraction; FS, fractional shortening. ${ }^{a} \mathrm{P}<0.05$ vs. sham group; ${ }^{b} \mathrm{P}<0.05$ vs. I/R group; ${ }^{\mathrm{C}} \mathrm{P}<0.05$ vs. $\mathrm{NRG}+\mathrm{I} / \mathrm{R}$ group. Data are presentedas the mean \pm standard deviation.

A
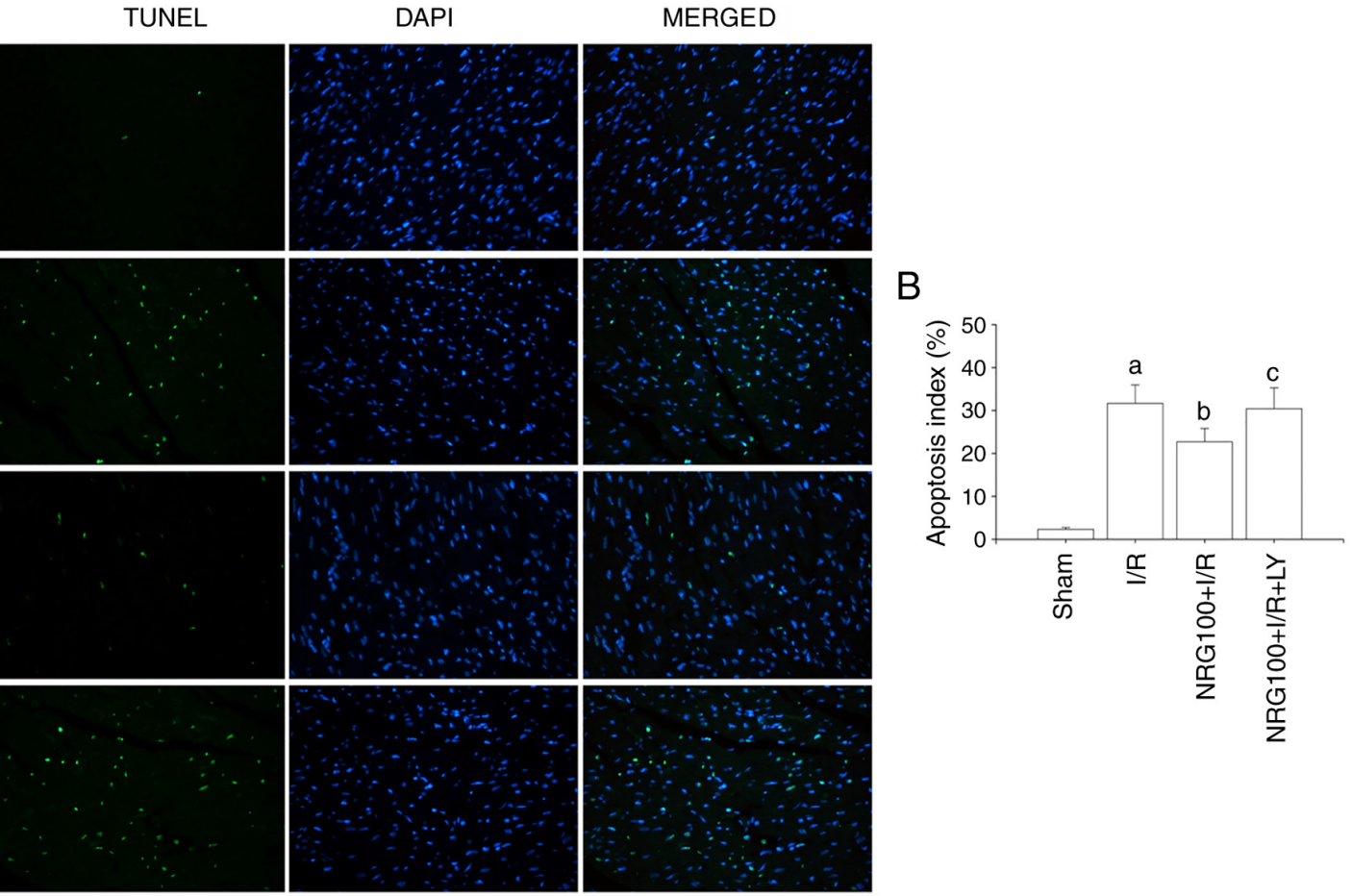

NRG100+l/R+LY
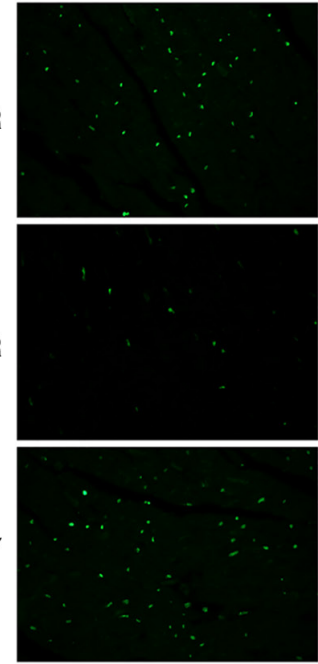

Figure 3. NRG pretreatment suppresses I/R-induced myocardial apoptosis. (A) Representative images of TUNEL stain; (B) Apoptosis index (n=5). NRG, naringin; I/R, ischemia reperfusion; LY, PI3K/Akt inhibitor LY294002; NRG100, $100 \mathrm{mg} / \mathrm{kg}$ naringin. ${ }^{\mathrm{a}} \mathrm{P}<0.05 \mathrm{vs}$. sham group; ${ }^{b} \mathrm{P}<0.05 \mathrm{vs}$. I/R group; ${ }^{\mathrm{c}} \mathrm{P}<0.05$ vs. NRG100 + I/R group.

These results suggested that NRG could suppress I/R induced myocardial apoptosis. However, LY294002 partially abolished the cardioprotective effects of NRG.

$N R G$ pretreatment activates the PI3K/Akt signaling pathway. As shown in Fig. 4A and E, I/R injury significantly reduced the phosphorylation of Akt as compared to sham group. However, NRG pretreatment remarkably promoted the phosphorylation of Akt. Administration of LY294002 abrogated the effect of NRG, indicating that NRG could activate the PI3K/Akt signaling pathway.

NRG pretreatment inhibits myocardial autophagy. Compared with the sham group, I/R significantly increased the expression of Beclin-1 and the LC3BII/LC3BI ratio (Fig. 4A, F and G). However, I/R-induced increases in Benlin-1 expression and
LC3BII/LC3BI ratio were suppressed by NRG pretreatment, suggesting that NRG could inhibit I/R-promoted myocardial autophagy. LY294002 partially abolished NRG-mediated anti-autophagy effect against I/R.

\section{Discussion}

The results of the present study demonstrated that NRG pretreatment could alleviate I/R-induced myocardial injury and cardiac dysfunction by suppressing apoptosis, the inflammatory response and oxidative stress. I/R-promoted myocardial autophagy was also inhibited by NRG pretreatment. Furthermore, the cardioprotective effects of NRG may be closely related to the activation of the PI3K/Akt signaling cascades.

NRG is a pharmacologically active constituent of tomentose pummelo peel, chemically known as 
A

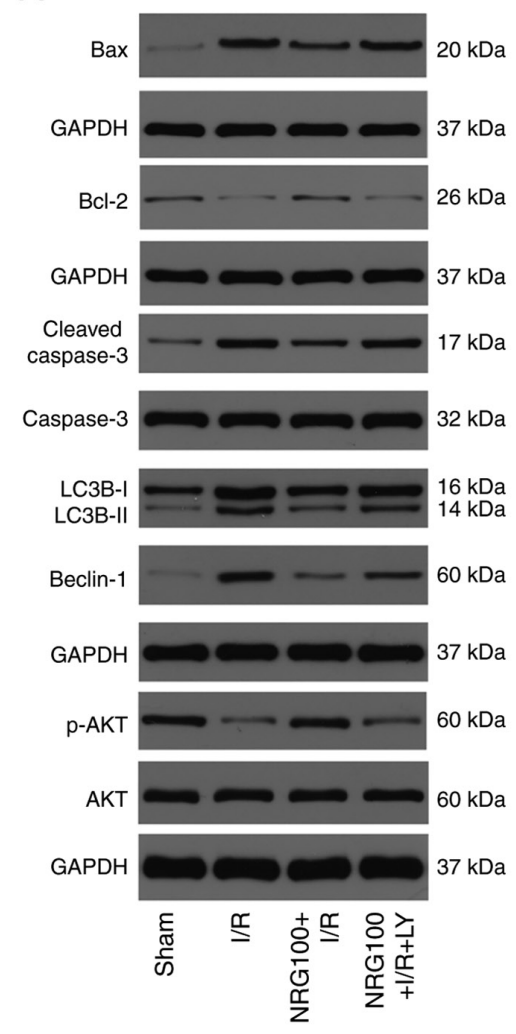

B

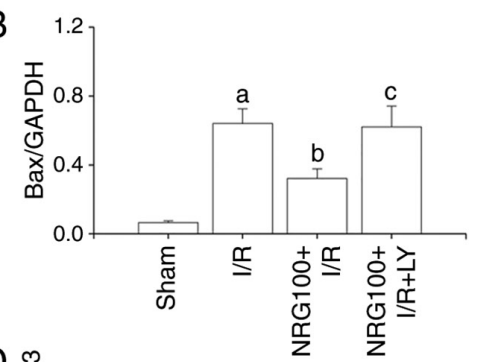

D

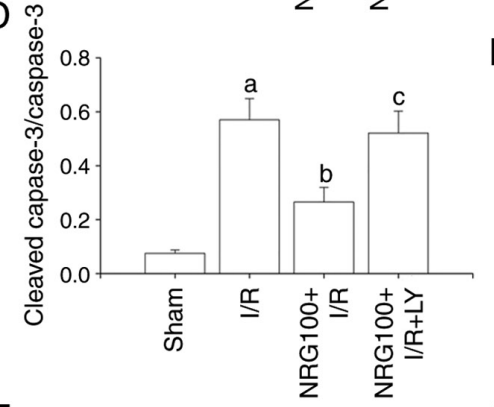

$F$

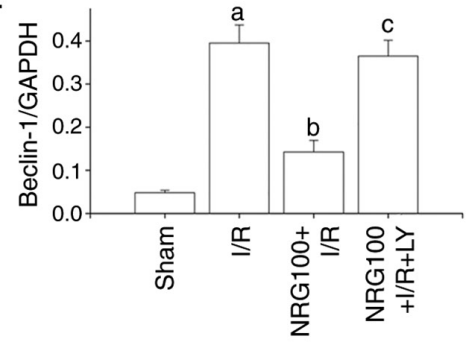

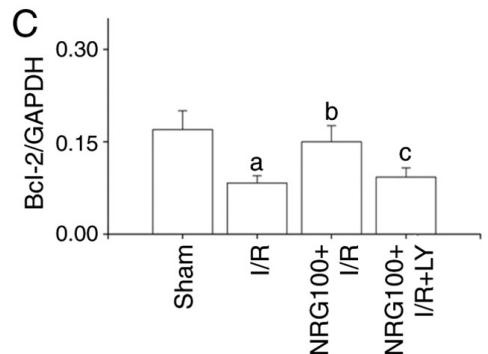

E
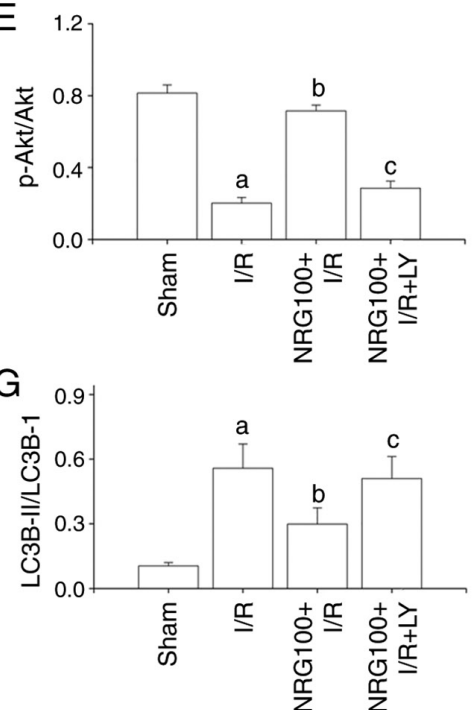

Figure 4. NRG pretreatment represses myocardial autophagy through PI3K/Akt signaling cascades. (A) Representative images of western blotting. Quantitative analysis of the (B) Bax, and (C) Bcl levels and the (D) cleaved caspase: caspase 3 and (E) p-Akt: Akt ratio and the (F) beclin-1 and (G) LC3BII: LC3BI ratio $(\mathrm{n}=3)$. NRG, naringin; I/R, ischemia reperfusion; LY, PI3K/Akt inhibitor LY294002; NRG100, $100 \mathrm{mg} / \mathrm{kg}$ naringin; p-Akt, phosphorylated Akt; LC3B, microtubule-associated protein $1 \mathrm{~B}$-light chain 3 . ${ }^{\mathrm{a}} \mathrm{P}<0.05$ vs. sham group; ${ }^{\mathrm{b}} \mathrm{P}<0.05 \mathrm{vs}$. I/R group; ${ }^{\mathrm{P}}<0.05 \mathrm{vs}$. NRG100 + I/R group.

4,5,7-trihydroxy-flavonone-7-rhamnogglucoside (5). NRG has been reported to have multiple cardioprotective effects. Rajadurai and Prince (21) demonstrated that NRG protected against isoproterenol-induced myocardial infarction by increasing the anti-oxidative capacity of the myocardium, as evidenced by the enhanced levels of SOD, glutathione s-transferase and glutathione peroxidase. Similar results were reported by Li et al (22) who revealed that NRG could suppress TNF- $\alpha$-induced oxidative stress and the inflammatory response via the PI3K/Akt pathway. I/R injury could significantly promote oxidative stress thus reduces the activity of $\operatorname{SOD}(23,24)$. It is known that Akt signaling plays a crucial role in the modulation of multiple biological processes in cardiovascular diseases. Numerous studies have demonstrated that pharmacological activation of PI3K/Akt could effectively enhance the activities of SOD against I/R injury (20,25). Yang et al (26) demonstrated that $\mathrm{PI} 3 \mathrm{~K} / \mathrm{Akt}$ cascades could facilitate Nrf2 nuclear translocation and therefore promote SOD activity in cardiomyocytes after hypoxia-reoxygenation procedures. Sun et al (27) found that PI3K/Akt signaling could notably activates the endothelial nitric oxide synthase pathway to enhance the anti-oxidative capacity of cardiomyocytes against I/R injury.

Myocardial I/R injury occurs with the restoration of blood flow to the occluded coronary artery (7). Myocardial apoptosis, the inflammatory response, oxidative stress and autophagy constitute crucial parts in the pathophysiological procedure of I/R injury (7). Rani et al (13). demonstrated that NRG significantly attenuated myocardial I/R injury by diminishing cardiac apoptosis as well as oxidative stress. A study by Chen et al (6) reported that NRG pretreatment mitigated anoxia/reoxygenation-induced apoptosis via the Nrf2 pathway in vitro. Consistent with these findings, the results of the present study also showed that NRG exerted cardioprotective effects against I/R injury via a reduction in the levels of pro-apoptotic proteins and oxidative stress, while promoting the expression of anti-apoptotic protein Bcl-2.

Myocardial I/R injury is also characterized by an excessive level of inflammation response (28). Inflammatory mediators such as IL-1 $\beta$, IL- 6 and TNF- $\alpha$ could significantly contribute to the myocardial dysfunction and structural alterations in the failing heart (29). Additionally, TNF- $\alpha$ could further exert deleterious effects on myocardial $\mathrm{I} / \mathrm{R}$ injury by promoting oxidative stress and the inflammatory response (30). Tong et al (20) demonstrated that I/R markedly increased the release of inflammatory cytokines, while the activation of the PI3K/Akt pathway could significantly repress I/R-mediated inflammation via down-regulation of high mobility group box protein. In sepsis-associated myocarditis, NRG also displayed anti-inflammatory effects (7). The above evidence supported the hypothesis that NRG could attenuate I/R injury via effectively inhibiting inflammatory response.

Autophagy is a dynamic process in which cellular waste is encapsulatedintodoublemembranevesiclesand thentransported to lysosomes for degradation (31). A normal state of autophagy 
is necessary for the renewal of organelles (32). The signaling pathways involved in autophagy have been extensively studied and the PI3K/Akt pathway is widely recognized as a crucial mediator in the regulation of autophagy $(33,34)$. Li et al $(35)$ demonstrated that pharmacological activation of PI3K/Akt could effectively suppress hypoxia/reoxygenation-induced autophagy in neonatal rats by targeting rapamycin. Ye et al (12) also confirmed that phosphorylated Akt could attenuate I/R-promoted myocardial autophagy via secretion of basic fibroblast growth factors. LC3BII/LC3BI ratio and Beclin-1 are representative autophagy markers, and their levels can directly reflect the degree of autophagy $(36,37)$. Consistent with the aforementioned studies, the results of the present study indicated that $\mathrm{I} / \mathrm{R}$ injury significantly promoted the expression of Beclin-1 and LC3BII/LC3BI ratio, while NRG pretreatment markedly reversed the up-regulated Beclin-1 and LC3BII/LC3BI ratio. However, these cardioprotective effects of NRG were partially abolished by the PI3K/Akt inhibitor LY294002. These results suggested that NRG may suppress I/R-induced myocardial autophagy by facilitating the PI3K/Akt signaling pathway.

There are a number of limitations to the present study. Specific PI3K/Akt inhibitors, such as Dactolisib or IC87114, might better have illustrated the cardioprotective role of NRG against I/R injury and an LY treatment alone group could also have aided the interpretation of the results.

In summary, the results of the present study indicated that NRG alleviated myocardial I/R injury via inhibition of cardiac apoptosis, inflammatory reactions and oxidative stress. In addition, NRG pretreatment also attenuated I/R-induced autophagy. The present data also suggested that such cardioprotective effects of NRG could be ascribed to the activation of the PI3K/Akt pathway. Furthermore, short-term NRG pretreatment may be a novel therapeutic strategy for the prevention of I/R injury in AMI patients.

\section{Acknowledgements}

Not applicable.

\section{Funding}

The present study was supported by a grant from the Natural Science Foundation of Hubei Province of China (grant no. 2015CFB223).

\section{Availability of data and materials}

The datasets used and/or analyzed during the current study are available from the corresponding author on reasonable request.

\section{Authors' contributions}

FL and ZZ were responsible for conceiving the study, performing the analysis and experiments and drafting the manuscript. JQ analyzed the data. CC and WY also performed the experiments. NW was involved in drafting the manuscript performing and designing the experiments as well as revising it for important intellectual content. FL and NW confirm the authenticity of all the raw data. All authors have read and approved the final manuscript.

\section{Ethics approval and consent participate}

Animal experiments were approved by the Institutional Animal Care and Use Committee of Hubei University of Medicine.

\section{Patient consent for publication}

Not applicable.

\section{Competing interest}

The authors declare that they have no competing interests.

\section{References}

1. Heusch G and Gersh BJ: The pathophysiology of acute myocardial infarction and strategies of protection beyond reperfusion: A continual challenge. Eur Heart J 38: 774-784, 2017.

2. Wang BF and Yoshioka J: The emerging role of thioredoxin-Interacting Protein in Myocardial Ischemia/Reperfusion Injury. J Cardiovasc Pharmacol Ther 22: 219-229, 2017.

3. Wang P, Sun J, Lv S, Xie T and Wang X: Apigenin alleviates myocardial reperfusion injury in rats by downregulating miR-15b. Med Sci Monit 25: 2764-2776, 2019.

4. Shen Y, Liu X, Shi J and Wu X: Involvement of Nrf2 in myocardial ischemia and reperfusion injury. Int J Biol Macromol 125: 496-502, 2019.

5. Jian CY, Ouyang HB, Xiang XH, Chen JL, Li YX, Zhou X, Wang JY, Yang Y, Zhong EY, Huang WH, et al: Naringin protects myocardial cells from doxorubicin induced apoptosis partially by inhibiting the p38MAPK pathway. Mol Med Rep 16: 9457-9463, 2017.

6. Chen RC, Sun GB, Wang J, Zhang HJ and Sun XB: Naringin protects against anoxia/reoxygenation-induced apoptosis in $\mathrm{H} 9 \mathrm{c} 2$ cells via the Nrf2 signaling pathway. Food Funct 6: 1331-1344, 2015.

7. Xianchu L, Lan PZ, Qiufang L, Yi L, Xiangcheng R, Wenqi H and Yang D: Naringin protects against lipopolysaccharide-induced cardiac injury in mice. Environ Toxicol Pharmacol 48: 1-6, 2016.

8. Li YP, Chen Z and Cai YH: Piperine protects against myocardial ischemia/reperfusion injury by activating the PI3K/AKT signaling pathway. Exp Ther Med 21: 374, 2021.

9. Zeng B, Liu L, Liao X, Zhang C and Ruan H: Thyroid hormone protects cardiomyocytes from $\mathrm{H} 2 \mathrm{O} 2$-induced oxidative stress via the PI3K-AKT signaling pathway. Exp Cell Res 380: 205-215, 2019.

10. Shaker ME, Ashamallah SA and Houssen ME: Celastrol ameliorates murine colitis via modulating oxidative stress, inflammatory cytokines and intestinal homeostasis. Chem Biol Interact 210: 26-33, 2014.

11. Wu S, Chang G, Gao L, Jiang D, Wang L, Li G, Luo X, Qin S, Guo $X$ and Zhang D: Trimetazidine protects against myocardial ischemia/reperfusion injury by inhibiting excessive autophagy. J Mol Med (Berl) 96: 791-806, 2018.

12. Ye G, Fu Q, Jiang L and Li Z: Vascular smooth muscle cells activate PI3K/Akt pathway to attenuate myocardial ischemia/reperfusion-induced apoptosis and autophagy by secreting bFGF. Biomed Pharmacother 107: 1779-1785, 2018.

13. Rani N, Bharti S, Manchanda M, Nag TC, Ray R, Chauhan SS, Kumari S and Arya DS: Regulation of heat shock proteins 27 and 70, p-Akt/p-eNOS and MAPKs by Naringin Dampens myocardial injury and dysfunction in vivo after ischemia/reperfusion. PLoS One 8: e82577, 2013.

14. Sherry EV: Review of the third edition of the Guide for the Care and Use of Agricultural Animals in Research and Teaching. J Am Assoc Lab Anim Sci, 51(3):298 300, 2012.

15. Cerkezkayabekir A, Sanal F, Bakar E, Ulucam E and Inan M: Naringin protects viscera from ischemia/reperfusion injury by regulating the nitric oxide level in a rat model. Biotech Histochem 92: 252-263, 2017 
16. Jiang X, Kong B, Shuai W, Shen C, Yang F, Fu H, Huang H, Jiang X, Kong B, Shuai W, et al: Loss of MD1 exacerbates myocardial ischemia/reperfusion injury and susceptibility to ventricular arrhythmia. Eur J Pharmacol 844: 79-86, 2019.

17. Lee JC and Sponenberg DP: Role of alpha 1-adrenoceptors in norepinephrine-induced cardiomyopathy. Am J Pathol 121: 316-321, 1985

18. Sepúlveda M, Gonano LA, Viotti M, Morell M, Blanco P López Alarcón M, Peroba Ramos I, Bastos Carvalho A, Medei E and Vila Petroff M: Calcium/Calmodulin protein kinase II-dependent ryanodine receptor phosphorylation mediates cardiac contractile dysfunction associated with sepsis. Crit Care Med 45: e399-e408, 2017.

19. Wang N, Zheng X, Qian J, Yao W, Bai L, Hou G, Qiu X, Li X and Jiang X: Renal sympathetic denervation alleviates myocardial fibrosis following isoproterenol-induced heart failure. Mol Med Rep 16: 5091-5098, 2017.

20. Tong S, Zhang L, Joseph J and Jiang X: Celastrol pretreatment attenuates rat myocardial ischemia/ reperfusion injury by inhibiting high mobility group box 1 protein expression via the PI3K/Akt pathway. Biochem Biophys Res Commun 497: 843-849, 2018.

21. Rajadurai M and Prince PS: Naringin ameliorates mitochondrial lipid peroxides, antioxidants and lipids in isoproterenol-induced myocardial infarction in Wistar rats. Phytother Res 23: 358-362, 2009.

22. Li W, Wang C, Peng J, Liang J, Jin Y, Liu Q, Meng Q, Liu K and Sun $\mathrm{H}$ : Naringin inhibits TNF- $\alpha$ induced oxidative stress and inflammatory response in HUVECs via Nox4/NF- $\kappa \mathrm{B}$ and PI3K/Akt pathways. Curr Pharm Biotechnol 15: 1173-1182, 2014

23. Wu Q, Lu K, Zhao Z, Wang B, Liu H, Zhang S, Liao J, Zeng Y, Dong Q, Zhao N, et al: Blockade of transient receptor potential vanilloid 4 enhances antioxidation after myocardial ischemia/reperfusion. Oxid Med Cell Longev 2019: 7283683, 2019.

24. Liu Z, Tao B, Fan S, Pu Y, Xia H and Xu L: MicroRNA 145 protects against myocardial ischemia reperfusion injury via CaMKII mediated anti apoptotic and anti inflammatory pathways. Oxid Med Cell Longev 2019: 8948657, 2019.

25. Dong J, Xu M, Zhang W and Che X: Effects of sevoflurane pretreatment on myocardial ischemia-reperfusion injury through the Akt/hypoxia-inducible factor 1-alpha (HIF-1 $\alpha$ )/vascular endothelial growth factor (VEGF) signaling pathway. Med Sci Monit 25: 3100-3107, 2019

26. Yang P, Zhou Y, Xia Q, Yao L and Chang X: Astragaloside IV regulates the $\mathrm{PI} 3 \mathrm{~K} / \mathrm{Akt} / \mathrm{HO}-1$ signaling pathway and inhibits $\mathrm{H} 9 \mathrm{c} 2$ cardiomyocyte injury induced by hypoxia-reoxygenation. Biol Pharm Bull 42: 721-727, 2019.
27. Sun Y, Jiang C, Jiang J and Qiu L: Dexmedetomidine protects mice against myocardium ischaemic/reperfusion injury by activating an AMPK/PI3K/Akt/eNOS pathway. Clin Exp Pharmacol Physiol 44: 946-953, 2017.

28. Liu S, He Y, Shi J, Liu L, Ma H, He L and Guo Y: Allicin attenuates myocardial ischemia reperfusion injury in rats by inhibition of inflammation and oxidative stress. Transplant Proc 51: 2060-2065, 2019.

29. Marchant DJ, Boyd JH, Lin DC, Granville DJ, Garmaroudi FS and McManus BM: Inflammation in myocardial diseases. Circ Res 110: 126-144, 2012.

30. Buja LM: Myocardial ischemia and reperfusion injury. Cardiovasc Pathol 14: 170-175, 2005.

31. Mizushima N and Komatsu M: Autophagy: Renovation of cells and tissues. Cell 147: 728-741, 2011.

32. Hang P, Zhao J, Su Z, Sun H, Chen T, Zhao L and Du Z: Choline inhibits ischemia-reperfusion-induced cardiomyocyte autophagy in rat myocardium by activating Akt/mTOR signaling. Cell Physiol Biochem 45: 2136-2144, 2018.

33. Shanware NP, Bray K and Abraham RT: The PI3K, metabolic, and autophagy networks: Interactive partners in cellular health and disease. Annu Rev Pharmacol Toxicol 53: 89-106, 2013.

34. Tang H, Song X, Ling Y, Wang X, Yang P, Luo T and Chen A: Puerarin attenuates myocardial hypoxia/reoxygenation injury by inhibiting autophagy via the Akt signaling pathway. Mol Med Rep 15: 3747-3754, 2017.

35. Li X, Zhu Q, Liu Y, Yang Z and Li B: Gastrodin protects myocardial cells against hypoxia/reoxygenation injury in neonatal rats by inhibiting cell autophagy through the activation of mTOR signals in PI3K-Akt pathway. J Pharm Pharmacol 70: 259-267, 2018.

36. Jiang P and Mizushima N: LC3- and p62-based biochemical methods for the analysis of autophagy progression in mammalian cells. Methods 75: 13-18, 2015

37. Zhou M, Zou YG, Xue YZ, Wang XH, Gao H, Dong HW and Zhang Q: Long non-coding RNA H19 protects acute myocardial infarction through activating autophagy in mice. Eur Rev Med Pharmacol Sci 22: 5647-5651, 2018. 